\title{
INJECTIVITY OF QUASI-ISOMETRIC MAPPINGS OF BALLS
}

\author{
JULIAN GEVIRTZ
}

\begin{abstract}
Let $X$ and $Y$ be real Banach spaces. A mapping $f$ of an open subset $R$ of $X$ into $Y$ is said to be $(m, M)$-isometric if it is a local homeomorphism for which $M \geq D^{+} f(x)$ and $m \leq D^{-} f(x)$ for all $x$ in $R$, where $D^{+} f(x)$ and $D^{-} f(x)$ are, respectively, the upper and lower limits of $|f(y)-f(x)| /|y-x|$ as $y \rightarrow x$. We show that if $R$ is a ball then all $(m, M)$ isometric mappings of $R$ are injective provided that $M / m<1.114 \ldots$ and we also give some numerical improvements of similar results of $F$. John for the special case that $X$ is a Hilbert space.
\end{abstract}

1. Introduction. In all that follows, $X$ and $Y$ denote real Banach spaces. Let $R$ be an open subset of $X$. Following John [1], a mapping $f: R \rightarrow Y$ is said to be $(m, M)$-isometric if it is a local homeomorphism (i.e., continuous, open and locally one-to-one) for which $M \geq D^{+} f(x)$ and $0<m \leq D^{-} f(x)$ for all $x$ in $R$, where $D^{+} f(x)$ and $D^{-} f(x)$ are, respectively, the upper and lower limits of $|f(y)-f(x)| /|y-x|$ as $y \rightarrow x$. Less precisely, $f$ is called quasi-isometric if it is $(m, M)$-isometric for some $m, M$. We cite two results of John as

THEOREM 1. Let $X$ be a Hilbert space and let $Y$ be a Banach space. Let $R \subset X$ be an open ball and let $f: R \rightarrow Y$ be an $(m, M)$-isometric mapping. Then either one of the following conditions implies that $f$ is one-to-one:

(A) $M / m<((1+\sqrt{5}) / 2)^{1 / 2}=1.272 \ldots($ see $[1])$,

(B) $Y$ is also a Hilbert space and $M / m<\sqrt{2}=1.414 \ldots$ (see [3]).

John's proofs of these injectivity criteria use properties of Hilbert spaces which have no analogue in general Banach spaces. The purpose of this paper is to show that even so it is possible to derive similar criteria valid in the more general context. We shall also show that the constant $((1+\sqrt{5}) / 2)^{1 / 2}$ of $(\mathrm{A})$ may be replaced by $\sqrt{2}$ and that the constant $\sqrt{2}$ of (B) may be replaced by $(1+\sqrt{2})^{1 / 2}=1.553 \ldots$ Let $\mu_{0}=1.114 \ldots$ denote the (unique) real root of the equation

$$
x=\frac{x+\left(25 x^{2}-8 x\right)^{1 / 2}}{2\left(3 x^{2}-x\right)}
$$

We shall prove

THEOREM 2. Let $X$ and $Y$ be Banach spaces. Let $R \subset X$ be an open ball and let $f: R \rightarrow Y$ be an $(m, M)$-isometric mapping. Then any one of the following conditions implies that $f$ is one-to-one:

(C) $M / m<\mu_{0}$,

(D) $X$ is a Hilbert space and $M / m<\sqrt{2}$,

(E) both $X$ and $Y$ are Hilbert spaces and $M / m<(1+\sqrt{2})^{1 / 2}$.

Received by the editors August 15, 1981.

1980 Mathematics Subject Classification. Primary 46B99, 47H99.

Key words and phrases. Quasi-isometric mapping, injectivity.

(c) 1982 American Mathematical Society $0002-9939 / 81 / 0000-0298 / \$ 02.25$ 
Our proof is a refinement of John's proofs of the sufficiency of conditions (A) and (B) but takes into account a number of other factors. It is, however, inherently simpler than his in that our approach allows us to work exclusively with quasiisometric homeomorphisms.

2. Preliminaries. As stated above, $X$ and $Y$ will always denote real Banach spaces. The open and closed balls with center $x$ and radius $r$ are denoted by $B(x, r)$ and $\bar{B}(x, r)$, respectively. For short we write $B(0, r)=B(r)$ and $\bar{B}(0, r)=\bar{B}(r)$. The boundary of a ball is indicated by the usual boundary symbol $\partial$. Our notation does not explicitly indicate to which space, $X$ or $Y$, a given ball belongs, but this will be clear from the context. As usual, $(x, y)$ and $[x, y]$ denote the open and closed straight line segments joining $x$ and $y$.

We now present some auxiliary results that will be used in the proof of Theorem 2. Lemmas 1 and 4 are taken from [2]. We include a proof of Lemma 2 since it does not appear there in explicit form. Lemma 5 is important to our approach since it is the basis of the reduction to homeomorphisms mentioned above.

LemMA 1 [2, Fundamental LemMa]. Let $A \subset X$ be convex and let $f: A \rightarrow$ $Y$ satisfy $D^{+} f(x) \leq M$ for all $x \in A$. Then $|f(p)-f(q)| \leq M|p-q|$ for all $p$, $q \in A$.

LEMMA 2. Let $A \subset X$ and let $f$ be a homeomorphism of $A$ into $Y$ which satisfies $D^{-} f(x) \geq m$ for all $x$ in $A$. If $p, q \in A$ and $[f(p), f(q)] \subset f(A)$, then $|f(p)-f(q)| \geq$ $m|p-q|$.

Proof. If $g$ denotes the restriction of $f^{-1}$ to $[f(p), f(q)]$, then $g$ satisfies $D^{+} g(x) \leq 1 / m$ for all $x$ in this interval. Thus, by Lemma 1 ,

$$
|p-q|=|g(f(p))-g(f(q))| \leq(1 / m)|f(p)-f(q)| .
$$

LeMMA 3 [2, TheOREM II]. Let $B(x, r)$ be a ball in $X$ and let $f: B(x, r) \rightarrow Y$ be a local homeomorphism which satisfies $D^{-} f(y) \geq m$ for all $y$ in $B(x, r)$. Then $f(B(x, r)) \supset B(f(x), m r)$.

LEMMA 4 [2, THEOREM III]. Let $B(x, r)$ be a ball in $X$ and let $f: B(x, r) \rightarrow Y$ be $(m, M)$-isometric. Then $m|p-q| \leq|f(p)-f(q)| \leq M|p-q|$ for all $p, q \in$ $B(x,(m / M) r)$.

LeMMA 5. Let $u>0$ and $B(u) \subset X$. Let $f: B(u) \rightarrow Y$ be an $(m, M)$-isometric mapping which is not one-to-one in $B(u)$. Then there are numbers $v, \eta$ with $u>$ $v>0$ and $\eta>0$ and sequences $\left\{a_{n}\right\}$ and $\left\{b_{n}\right\}$ of points in $B(u)$ such that

(i) $f$ is a homeomorphism on $B(v)$,

(ii) $f\left(a_{n}\right)=f\left(b_{n}\right)$ for all $n$,

(iii) $\left|a_{n}-b_{n}\right| \geq \eta$ for all $n$, and

(iv) $\left|a_{n}\right| \rightarrow v$ and $\left|b_{n}\right| \rightarrow v$ as $n \rightarrow \infty$.

Proof. Let $v=\inf \{r: f$ is not one-to-one in $B(r)\}$. We have $u>v>0$ since $f$ is a homeomorphism in a neighborhood of 0 . Let $\delta=(u-v) / 2$. For each integer $n \geq 1$ there exist two distinct points $a_{n}$ and $b_{n}$ in $B(v+\delta)$ for which $\left|a_{n}\right|$, $\left|b_{n}\right|<v+1 / n$ and $f\left(a_{n}\right)=f\left(b_{n}\right)$. Since $B(x, \delta) \subset B(u)$ for $x \in B(v+\delta)$, we see by Lemma 4 that $\left|a_{n}-b_{n}\right| \geq \eta=(m / M) \delta$. Selecting subsequences of $\left\{a_{n}\right\}$ and 
$\left\{b_{n}\right\}$, we may assume that $\left|a_{n}\right|$ and $\left|b_{n}\right|$ converge to numbers $\alpha$ and $\beta$, respectively. For the $v, \eta,\left\{a_{n}\right\}$ and $\left\{b_{n}\right\}$ that we have found, we have (i), (ii) and (iii). Thus, to finish we have only to show that $\alpha=\beta=v$.

Obviously $\alpha, \beta \leq v$. Assume that $\alpha<v$. Let $\xi=\min \{(v-\alpha) / 2, \eta / 2\}$ and $T=f\left(B\left(a_{n}, \xi\right) \cap B(v)\right) \cap f\left(B\left(b_{n}, \xi\right) \cap B(v)\right)$. On one hand, we have $T=\emptyset$, since $B\left(a_{n}, \xi\right) \cap B\left(b_{n}, \xi\right)=\emptyset$ and $f$ is one-to-one on $B(v)$. However, there is an $N_{1}$ such that $B\left(a_{n}, \xi\right) \subset B(v)$ for $n \geq N_{1}$, since $\left|a_{n}\right| \rightarrow \alpha$, and there is an $N_{2}$ such that $W=B\left(b_{n},(m / M) \xi\right) \cap B(v) \neq \emptyset$ for $n \geq N_{2}$, since $\left|b_{n}\right| \rightarrow \beta \leq v$. By Lemmas 1 and $3, f(W) \subset B\left(f\left(b_{n}\right), m \xi\right)=B\left(f\left(a_{n}\right), m \xi\right) \subset f\left(B\left(a_{n}, \xi\right)\right)=f\left(B\left(a_{n}, \xi\right) \cap B(v)\right)$ for $n \geq N_{1}$. Since $W \subset B\left(b_{n}, \xi\right) \cap B(v)$, we have $T \supset f(W) \neq \emptyset$ for $n \geq N_{1}, N_{2}$, which is a contradiction. Thus $\alpha=v$ and the same argument with $\left\{a_{n}\right\}$ and $\left\{b_{n}\right\}$ interchanged shows that $\beta=v$. This finishes the proof.

3. Proof of Theorem 2. The proof is divided into three parts. Parts II and III depend on Part I but are independent of each other. In Part II we establish the sufficiency of condition (C), and in the final part we deal with (D) and (E).

I. Let $f: R \rightarrow Y$ be $(m, M)$-isometric and let $\mu=M / m$. We assume here that $f$ is noninjective and in Parts II and III we show that this leads to lower bounds for $\mu$. Clearly, we may assume that 0 is the center of $R$, that is, $R=B(u)$ for some $u>0$. By Lemma 5 there exist $v, 0<v<u$, and $\eta>0$ such that $f$ is one-to-one in $B(v)$, and for all $\epsilon>0$ there exist points $a^{\prime}$ and $b^{\prime}$ in $B(u) \backslash\{0\}$ for which $\left|a^{\prime}-b^{\prime}\right| \geq \eta,|| a^{\prime}|-v|<\epsilon,|| b^{\prime}|-v|<\epsilon$ and $f\left(a^{\prime}\right)=f\left(b^{\prime}\right)$. By considering $g$ defined by $g(x)=f(v x)$ instead of $f$, we may assume that $v=1$. Henceforth $B(v)=B(1)$ shall be denoted by $B$.

Let $\bar{a}=a^{\prime} /\left|a^{\prime}\right|$ and $\bar{b}=b^{\prime} /\left|b^{\prime}\right|$. We have

$$
\left|\bar{a}-a^{\prime}\right|,\left|\bar{b}-b^{\prime}\right|<\epsilon .
$$

We consider numbers $t$ and $\epsilon$ which satisfy

$$
\frac{2 \mu(1+\epsilon)}{2 \mu+\eta-2 \epsilon}<\frac{4 \mu+\eta}{4 \mu+2 \eta}<t<1 ; \quad 0<\epsilon<\eta / 2
$$

The reason for imposing these conditions will become clear later on. We consider $\tau \in(0, t)$. Since $0=\left|f\left(a^{\prime}\right)-f\left(b^{\prime}\right)\right| \geq|f(\tau \bar{a})-f(\tau \bar{b})|-\left|f\left(a^{\prime}\right)-f(\tau \bar{a})\right|-\mid f\left(b^{\prime}\right)-$ $f(\tau \bar{b}) \mid$, we have, by $(2)$ and Lemma 1 , that

$$
|f(\tau \bar{a})-f(\tau \bar{b})| \leq 2 M(1-\tau+\epsilon) .
$$

For $\tau$ sufficiently close to 0 , there holds

$$
[f(\tau \bar{a}), f(\tau \bar{b})] \subset f(B(t))
$$

since $f(B(t))$ is open and contains $f(0)$. If (5) is true for a given $\tau$, then by Lemma 2 we have $|f(\tau \bar{a})-f(\tau \bar{b})| \geq m \tau|\bar{a}-\bar{b}|$, and so by $(4), 2 M(1-\tau+\epsilon) \geq m \tau(\eta-2 \epsilon)$, because $|\bar{a}-\bar{b}| \geq \eta-2 \epsilon$, by (2). Hence (5) implies $\tau \leq 2 \mu(1+\epsilon) /(2 \mu+\eta-2 \epsilon)$. Thus $s=\sup \{\tau>0$ : line (5) holds $\}$ satisfies

$$
0<s \leq \frac{4 \mu+\eta}{4 \mu+2 \eta}<t
$$

by the condition (3) that we have imposed on $\epsilon$ and $t$. From now on $s \bar{a}=a$ and $s \bar{b}=b$. By continuity we have $[f(a), f(b)] \subset f(\bar{B}(t)) \subset f(B)$ and $[f(a), f(b)] \cap$ $f(\partial B(t)) \neq \emptyset$. Thus there is a point $c \in \partial B(t)$ for which $f(c) \in(f(a), f(b))$. Since 
$|f(a)-f(b)|=|f(a)-f(c)|+|f(b)-f(c)|$, Lemma 2 implies

$$
|f(a)-f(b)| \geq m(|a-c|+|b-c|) \text {. }
$$

II. Condition (C). Since $|a|=|b|=s$ and $|c|=t>s$, we have $|a-c|,|b-c| \geq$ $t-s$. We define $\lambda \geq 1$ by

$$
|a-c|+|b-c|=2 \lambda(t-s) \text {. }
$$

Line (4), with $\tau=s$, together with lines (7) and (8) yields

$$
\mu \geq \frac{\lambda(t-s)}{1-s+\epsilon} \text {. }
$$

Since this is a usable lower bound for $\mu$ only when $\lambda$ stays away from 1 , we now find another bound that works well for $\lambda$ near 1 . Let $w=(a+b) / 2$. By (8) and the fact that $w \in \bar{B}(s)$ and $c \in \partial B(t)$, we have

$$
t-s \leq|w-c| \leq \lambda(t-s)
$$

Since $B(w, 1-s) \subset B$, Lemma 4 implies that $|f(p)-f(q)| \geq m|p-q|$ for all $p$, $q$ in $B(w,(1-s) / \mu)$. Let $c^{\prime}=w+(1 / \lambda \mu)(c-w)$ and $c^{\prime \prime}=w-(1 / \lambda \mu)(c-w)$. Line (10) implies that $c^{\prime}, c^{\prime \prime} \in B(w,(1-s) / \mu)$ and that $\left|c^{\prime \prime}-c^{\prime}\right| \geq 2(t-s) / \lambda \mu$. Hence, $\left|f\left(c^{\prime \prime}\right)-f\left(c^{\prime}\right)\right| \geq(2 m / \lambda \mu)(t-s)$. Since $\left|f\left(c^{\prime \prime}\right)-f(c)\right| \geq\left|f\left(c^{\prime \prime}\right)-f\left(c^{\prime}\right)\right|-$ $\left|f\left(c^{\prime}\right)-f(c)\right|$, this, together with (10), implies that $\left|f\left(c^{\prime \prime}\right)-f(c)\right|$ is bounded below by $L=(2 m / \lambda \mu)(t-s)-M(1-1 / \lambda \mu) \lambda(t-s)$. On the other hand, by (8), $|a-w|=|b-w|=|a-b| / 2 \leq \lambda(t-s) \leq(2 \lambda-1)(t-s)$ and $|a-(2 w-c)|=$ $|b-c| \leq(2 \lambda-1)(t-s)$. Analogously, $|b-(2 w-c)| \leq(2 \lambda-1)(t-s)$. Since $c^{\prime \prime} \in[w, 2 w-c]$, we conclude that $\left|c^{\prime \prime}-a\right|,\left|c^{\prime \prime}-b\right| \leq(2 \lambda-1)(t-s)$. But $f(c) \in[f(a), f(b)]$ and so

$$
\begin{aligned}
\left|f\left(c^{\prime \prime}\right)-f(c)\right| & \leq \max \left\{\left|f\left(c^{\prime \prime}\right)-f(a)\right|,\left|f\left(c^{\prime \prime}\right)-f(b)\right|\right\} \\
& \leq M\left(\max \left\{\left|c^{\prime \prime}-a\right|,\left|c^{\prime \prime}-b\right|\right\}\right) \leq M(2 \lambda-1)(t-s),
\end{aligned}
$$

by Lemma 1 . Hence, $M(2 \lambda-1)(t-s) \geq L$. Since the conditions (3) on $t$ and $\epsilon$ permit them to approach 1 and 0 , respectively, and since (6) implies that $s$ is bounded away from 1, a simple compactness argument applied to (9) and this last inequality shows that there is a number $\lambda \geq 1$ for which

$$
\mu \geq \lambda \text { and }\left(3 \lambda^{2}-\lambda\right) \mu^{2}-\lambda \mu-2 \geq 0 \text {. }
$$

The second of these two inequalities implies that $\mu$ is bounded below by $\left(\lambda+\left(25 \lambda^{2}-8 \lambda\right)^{1 / 2}\right) /\left(2\left(3 \lambda^{2}-\lambda\right)\right)$. Since this function is decreasing for $\lambda \geq 1,(11)$ leads us to conclude that if $f$ is not one-to-one, then $\mu \geq \mu_{0}$, where $\mu_{0}=1.114 \ldots$ is the root of (1). Thus (C) implies that $f$ is injective.

III. Conditions (D) and (E). We now assume that $X$ is a Hilbert space. Since $|a|=|b|=s$ and $|c|=t>s$, simple geometric arguments (based on the fact that $c$ does not lie in an $r$-neighborhood of $[a, b])$ show that

$$
|a-c|+|b-c| \geq 2\left(r^{2}+d^{2}\right)^{1 / 2}
$$

where $r=t-s$ and $d=|a-b| / 2$. Line (4) with $\tau=s$ together with lines (7) and (12) implies that

$$
\mu \geq \frac{t-s}{1-s+\epsilon}\left(1+(d / r)^{2}\right)^{1 / 2}
$$


However, Lemma 1 implies that $M|a-b| \geq|f(a)-f(b)|$, which together with (7) ind (12) gives

$$
\mu \geq\left(1+(r / d)^{2}\right)^{1 / 2}>\frac{t-s}{1-s+\epsilon}\left(1+(r / d)^{2}\right)^{1 / 2} .
$$

If we assume that $Y$ is also a Hilbert space, we can improve (14D) as follows. Since $f(c) \in(f(a), f(b))$ and the simple arc $f([a, b])$ connects $f(a)$ to $f(b)$, there is a point $e \in(a, b)$ for which $[f(e), f(c)]$ is perpendicular to $[f(a), f(b)]$. Since $|e|<s$ and $|c|=t$, we have $c \notin B(e, t-s)$ and since $f$ is one-to-one in $B$, we have $f(c) \notin$ $f(B(e, t-s)) \supset B(f(e), m(t-s))$, by Lemma 3. Thus $|f(e)-f(c)| \geq m(t-s)=$ $n r$. Elementary plane geometry and Lemma 1 then imply

$$
\begin{aligned}
M|a-b| & =M(|a-e|+|b-e|) \geq|f(a)-f(e)|+|f(b)-f(e)| \\
& \geq 2\left((|f(a)-f(b)| / 2)^{2}+|f(e)-f(c)|^{2}\right)^{1 / 2} \\
& \geq 2\left((|f(a)-f(b)| / 2)^{2}+m^{2} r^{2}\right)^{1 / 2} .
\end{aligned}
$$

Hence, (7) and (12) now give

$$
\mu \geq\left(1+2(r / d)^{2}\right)^{1 / 2}>\frac{t-s}{1-s+\epsilon}\left(1+2(r / d)^{2}\right)^{1 / 2} .
$$

Line (13), together with lines (14D) and (14E), respectively, implies

$$
\mu \geq \sqrt{2}(t-s) /(1-s+\epsilon) \text { and } \mu \geq(1+\sqrt{2})^{1 / 2}(t-s) /(1-s+\epsilon) \text {. }
$$

Since the conditions (3) imposed on $t$ and $\epsilon$ allow them to approach 1 and 0 , respectively, and since by (6), $s$ is bounded away from 1 , we conclude that if $f: R \rightarrow$ $Y$ is not one-to-one, then $\mu \geq \sqrt{2}$ in the case that $X$ is a Hilbert space and $\mu \geq$ $(1+\sqrt{2})^{1 / 2}$ in the case that both $X$ and $Y$ are Hilbert spaces. Thus, each of the conditions (D) and (E) implies that $f$ is injective.

4. Remarks. (i) Using a modification of the argument of Part III of the proof of Theorem 2, it is possible to show that we may replace $(1+\sqrt{2})^{1 / 2}$ in $(\mathrm{E})$ by the minimum for $x \geq 0$ of the function $\left(\pi+2\left(1+x^{2}\right)^{1 / 2}\right) /(1+\pi / 2+x)$, which is approximately 1.657 . The details will be given elsewhere.

(ii) It is probable that a more careful analysis than the one given here would result in a value of $\mu_{0}$ greater than 1.114 and it would be of interest to see just how large a value can be derived by the kind of argument we have used. (The best constant cannot exceed 2 , as can be shown by trivial examples.)

\section{REFERENCES}

1. F. John, Quasi-isometric mappings in Hilbert space, New York Univ., Courant Inst. Math. Sci., Res. Rep. No. IMM-NYU 336, 1965.

2. __ On quasi-isometric mappings, I, Comm. Pure Appl. Math. 21 (1968), 77-110.

3. ___ On quasi-isometric mappings, II, Comm. Pure Appl. Math. 22 (1969), 265-278.

Instituto de Matemática, Pontificia Universidad Católica de Chile, CAsilla 114-D, SANTiago, Chile 